\title{
Article \\ Construction of an Explicit Solution of a Time-Fractional Multidimensional Differential Equation
}

\author{
Murat A. Sultanov ${ }^{1, *\left(\mathbb{D}, \text { Durdimurod K. Durdiev }^{2} \text { and Askar A. Rahmonov }\right.}{ }^{3}$ De $^{(}$ \\ 1 Department of Mathematics, Faculty of Natural Science, Khoja Akhmet Yassawi International \\ Kazakh-Turkish University, Turkistan 160200, Kazakhstan \\ 2 Bukhara Branch of the Institute of Mathematics, Academy of Sciences of the Republic of Uzbekistan, \\ Bukhara 100170, Uzbekistan; durdiev65@mail.ru \\ 3 Department of Mathematics, Bukhara State University, Bukhara 200114, Uzbekistan; araxmonov@mail.ru \\ * Correspondence: murat.sultanov@ayu.edu.kz
}

check for updates

Citation: Sultanov, M.A.; Durdiev, D.K.; Rahmonov, A.A. Construction of an Explicit Solution of a Time-Fractional Multidimensional Differential Equation. Mathematics 2021, 9, 2052. https://doi.org/ $10.3390 /$ math9172052

Academic Editor: Ivanka Stamova

Received: 13 July 2021

Accepted: 19 August 2021

Published: 26 August 2021

Publisher's Note: MDPI stays neutral with regard to jurisdictional claims in published maps and institutional affiliations.

Copyright: (c) 2021 by the authors. Licensee MDPI, Basel, Switzerland. This article is an open access article distributed under the terms and conditions of the Creative Commons Attribution (CC BY) license (https:/ / creativecommons.org/licenses/by/ $4.0 /)$.

\begin{abstract}
In this work, an explicit solution of the initial-boundary value problem for a multidimensional time-fractional differential equation is constructed. The possibility of obtaining this equation from an integro-differential wave equation with a Mittag-Leffler-type memory kernel is shown. An explicit solution to the problem under consideration is obtained using the Laplace and Fourier transforms, the properties of the Fox $H$-functions and the convolution theorem.
\end{abstract}

Keywords: time-fractional equation; Fox function; Hankel transform; Laplace operator; Green function; exact solution

MSC: 35C15; 35L15

\section{Introduction to the Problem and Its Setting}

The investigation of many mathematical models that have a fractal structure have numerous applied applications. The presence of memory in such models indicates the dependence of its current state on a finite number of its previous states. This means the non-local properties of non-classical mathematical models, for example, in the mechanics of viscoelastic media when describing the action of aftereffect $[1,2]$. In materials science, material fatigue exists, which leads to the destruction of the material [3], in mathematical models of economics, the effect of dynamic memory is associated with the principle of causation of economic models [4].

As shown in [5], the memory property can be described using the mathematical apparatus of fractional calculus or using the operators fractional derivative. Fractional derivative operators have many definitions and have unique properties, but they all describe to one degree or another memory effect characterizing information about the previous states of the system.

The mathematical apparatus for describing hereditary mechanics is the apparatus of integro-differential equations with a convolution integral terms, in which kernels are called functions of memory [6]. In [7-22], a wide class of inverse problems of determining these kernels from hyperbolic and parabolic integro-differential equations was studied on the basis of an additional condition on the solution of the direct problem.

If the memory functions are given and are power-law, then we can go to other types of equations that are based on derivatives of fractional orders, properties of which are considered in table books on fractional calculus [23]. Processes and systems that are described using fractional derivatives orders are called fractal; for example, oscillators are called fractal in the theory of hereditary fluctuations [24].

The unique solvability of Cauchy and initial-boundary value problems for different types of FDE (fractional differential equations) were analyzed in the works [25-38]. 
To solve initial-boundary value problems for fractional differential equations of diffusion type, various methods are used: the Green's function method, methods based on the integral transforms of Fourier, Laplace and Mellin, the method of separation of variables, methods of reduction to integral equations of the Volterra type, and other methods. At the same time, there are relatively few methods for obtaining explicit solutions of fractional differential equations.

In this paper, we consider the following $n$-dimensional differential equation with Caputo fractional derivative of order $\alpha>0$

$$
\rho \frac{\partial^{m} u(x, t)}{\partial t^{m}}+{ }_{0}^{C} D_{t}^{\alpha} u-\Delta u(x, t)=f(x, t),
$$

where the initial and boundary conditions are:

$$
\left.\frac{\partial^{j} u}{\partial t^{j}}\right|_{t=0}=\varphi_{j}(x), j=0,1, \ldots, m-1, \quad \lim _{|x| \rightarrow \infty}(u, \nabla u)(x, t)=0, \quad t>0, x \in \mathbb{R}^{n}
$$

here, the $\rho$-positive constant, $f(x, t) \in L_{1}\left(\mathbb{R}^{n}\right) \times L_{1}(0, \infty)$ and $\varphi_{j}(x) \in L_{1}\left(\mathbb{R}^{n}\right)$ are given, ${ }_{0}^{C} D_{t}^{\alpha}$ is defined for $m-1<\alpha \leq m$ ( $m$ is a positive integer) as in [23]

$$
\begin{gathered}
{ }_{0}^{C} D_{t}^{\alpha} u(x, t):=\frac{1}{\Gamma(m-\alpha)} \int_{0}^{t} \frac{\partial^{m} u(x, \tau)}{\partial \tau^{m}} \frac{d \tau}{(t-\tau)^{\alpha+1-m}}, m-1<\alpha<m, \\
{ }_{0}^{C} D_{t}^{\alpha} u(x, t):=\frac{\partial^{m} u(x, t)}{\partial t^{m}}, \alpha=m ;
\end{gathered}
$$

$\Delta$ is the $n$-dimensional Laplace operator with respect to $x$ and $\nabla=\left(\frac{\partial}{\partial x_{1}}, \ldots, \frac{\partial}{\partial x_{n}}\right)$.

Equation (1) for $\rho=1, m=1$ describes the anomalously diffusive transport of solute in heterogeneous porous media [27]. This equation containing both the classical and fractional derivatives is more general and is of interest in the theory of the differential equations with fractional derivatives.

Our main goal is to obtain an explicit formula that gives a solution to the problem (1) and (2).

\section{General Theories}

In this section, we recall basic definitions and notations from integral transforms, special functions and fractional calculus.

Mittag-Leffler function. The Mittag-Leffler functions are defined as follows:

$$
E_{\alpha}(z):=\sum_{n=0}^{\infty} \frac{z^{n}}{\Gamma(\alpha n+1)}=: E_{\alpha, 1}(z) \quad \text { and } \quad E_{\alpha, \beta}(z):=\sum_{n=0}^{\infty} \frac{z^{n}}{\Gamma(\alpha n+\beta)},
$$

respectively, where $\alpha, z, \beta \in \mathbb{C} ; \mathfrak{R}(\alpha)>0, \mathfrak{R}(\alpha)$ denotes the real part of the complex number $\alpha$.

The Prabhakar function is [36]:

$$
E_{\alpha, \beta}^{\gamma}:=\sum_{n=0}^{\infty} \frac{(\gamma)_{n}}{\Gamma(\alpha n+\beta)} \frac{z^{n}}{n !}
$$

where $\alpha, \beta, \gamma, z \in \mathbb{C}$, and $(\gamma)_{n}$ denotes the Pochammer symbol or the shifted factorial determined by

$$
(\gamma)_{0}=1, \quad(\gamma)_{n}=\gamma(\gamma+1) \ldots(\gamma+n-1), \quad \gamma \neq 0
$$

Moreover, we can write $(\gamma)_{n}=\Gamma(\gamma+n) / \Gamma(\gamma)$, where $\Gamma(\gamma)$ is the usual Gamma function. We have running special cases: $E_{\alpha, \beta}^{1}(z)=E_{\alpha, \beta}(z)$ and $E_{\alpha, 1}^{1}=E_{\alpha}(z)$. 
The Mittag-Leffler function has numerous applications, and many authors have generalized it. In this category is the work of Haubold et al. [37].

Fox's $H$-function. Fox's $H$ - function is a special function of fractional calculus and contains Mittag-Leffler functions. This function was introduced by Fox [38] as a generalization of the Meyer function. Here we give the definition and properties of this function [29] which we need, with minor changes in the notation.

The $H$-function is defined by means of a Mellin-Barnes-type integral in the following manner:

$$
H_{p, q}^{m, n}\left[\left.z\right|_{\left(b_{1}, B_{1}\right), \ldots,\left(b_{q}, B_{q}\right)} ^{\left(a_{1}, A_{1}\right), \ldots,\left(a_{p}, A_{p}\right)}\right]=H_{p, q}^{m, n}\left[\left.z\right|_{\left(b_{q}, B_{q}\right)} ^{\left(p, A_{p}\right)}\right]=\frac{1}{2 \pi i} \int_{\Omega} \Theta(s) z^{-s} d s,
$$

where the integrand is

$$
\Theta(s)=\frac{\prod_{j=1}^{m} \Gamma\left(b_{j}+B_{j} s\right) \prod_{k=1}^{n} \Gamma\left(1-a_{k}-A_{k} s\right)}{\prod_{j=m+1}^{q} \Gamma\left(1-b_{j}-B_{j} s\right) \prod_{k=n+1}^{p} \Gamma\left(a_{k}+A_{k} s\right)},
$$

here $i=(-1)^{1 / 2}, z \neq 0, z^{-s}=\exp [-s\{\ln |z|+i \arg z\}]$.

In (2.3), an empty product is always interpreted as unity, $m, n, p, q \in \mathbb{N}_{0}$, with $0 \leq n \leq p, 1 \leq m \leq q, A_{k}, B_{k} \in \mathbb{R}^{+}:=(0,+\infty),{ }_{k}, b_{j} \in \mathbb{R}(\mathbb{C}), k=1, \ldots, p, j=1, \ldots, q$.

The contour $\Omega$ starting at the point $\sigma-i \infty$ and going to $\sigma+i \infty, \sigma \in \mathbb{R}$ such that all the poles of $\Gamma\left(b_{j}+B_{j} s\right), j=1, \ldots, m$, are separated from those of $\Gamma\left(1-a_{k}-A_{k} s\right), k=1, \ldots, n$.

The conditions for the convergence of the integral are given below:

(1) $\alpha>0,|\arg z|<\frac{1}{2} \pi \alpha$ and $z \neq 0$;

where

(2) $\alpha=0, \sigma \mu+\mathfrak{R}(\delta)<-1, \arg z=0$ and $z \neq 0$

$$
\begin{gathered}
\alpha:=\sum_{j=1}^{n} A_{j}-\sum_{j=n+1}^{p} A_{j}+\sum_{j=1}^{m} B_{j}-\sum_{j=m+1}^{q} B_{j}, \\
\mu:=\sum_{j=1}^{q} B_{j}-\sum_{j=1}^{p} A_{j}, \quad \delta:=\sum_{j=1}^{q} b_{j}-\sum_{j=1}^{p} a_{j}+\frac{p-q}{2} .
\end{gathered}
$$

The $H$ - function is studied in more detail in [29]. We only mentioned some properties and its Hankel transform that will be used in this paper.

Properties of $H$-function. ([29], pp. 11-13) We have the following reduction formulae:

$$
\text { - } \quad H_{p, q}^{m, n}\left[\left.z\right|_{\left(b_{1}, B_{1}\right), \ldots,\left(b_{q}, B_{q}\right)} ^{\left(1, A_{1}\right), \ldots,\left(p_{p-1}, A_{p-1}\right),\left(b_{1}, B_{1}\right)}\right]=H_{p-1, q-1}^{m-1, n}\left[\left.z\right|_{\left(b_{2}, B_{2}\right), \ldots,\left(b_{q}, B_{q}\right)} ^{\left(1, A_{1}\right), \ldots,\left(p-1, A_{p-1}\right)}\right],
$$

provided $m \geq 1$ and $p>n$.

$$
\begin{aligned}
& \text { - } H_{p, q}^{m, n}\left[\left.z\right|_{\left(b_{q}, B_{q}\right)} ^{\left(p, A_{p}\right)}\right]=H_{q, p}^{n, m}\left[\left.\frac{1}{z}\right|_{\left(1-p, A_{p}\right)} ^{\left(1-b_{q}, B_{q}\right)}\right], \\
& \text { - } \quad H_{p, q}^{m, n}\left[\left.z\right|_{\left(b_{q}, B_{q}\right)} ^{\left({ }_{p}, A_{p}\right)}\right]=k H_{p, q}^{m, n}\left[\left.z^{k}\right|_{\left(k a_{p}, A_{p}\right)} ^{\left(b_{q}, k B_{q}\right)}\right], \quad k>0 .
\end{aligned}
$$

Hankel Transform of the $H$-function. ([29], p. 57) $H_{v}\{f(x) ; \lambda\}:$ Hankel transform of order $v$ of $f(x)$. 
Definition 1. The Hankel transform of a function $f(x)$ is defined as follows

$$
g(\lambda)=\int_{0}^{\infty}(\lambda x)^{\frac{1}{2}} J_{v}(\lambda x) f(x) d x, \quad \lambda>0
$$

The inverse Hankel transform is given by

$$
f(x)=\int_{0}^{\infty}(x \lambda)^{\frac{1}{2}} J_{v}(x \lambda) g(\lambda) d \lambda, \quad \mathfrak{R}(v)>-1 .
$$

where $J_{v}(z)$ is a Bessel function of the first kind and defined by

$$
J_{v}(z)=\sum_{k=0}^{\infty} \frac{(-1)^{k}}{\Gamma(1+v+k) k !}\left(\frac{z}{2}\right)^{v+2 k}
$$

Suppose that $\alpha>0$ or $\alpha=\mu=0$ and $\mathfrak{R}(\delta)<-1$. Then if $\lambda, v, b \in \mathbb{C}, \sigma>0$ satisfy the conditions

$$
\mathfrak{R}(\lambda)+\mathfrak{R}(v)+\sigma \min _{1 \leq j \leq m}\left[\frac{\mathfrak{R}\left(b_{j}\right)}{B_{j}}\right]>-1
$$

and

$$
\mathfrak{R}(\lambda)+\sigma \min _{1 \leq j \leq m}\left[\frac{1}{A_{j}}-\frac{\mathfrak{R}\left({ }_{j}\right)}{A_{j}}\right]<\frac{3}{2}
$$

for $\mathfrak{R}(v)>-\frac{1}{2}$. Then for,$b>0$ there holds the formula

$$
\begin{gathered}
\int_{0}^{\infty} x^{\lambda-1} J_{v}(a x) H_{p, q}^{m, n}\left[\left.b x^{\sigma}\right|_{\left(b_{q}, B_{q}\right)} ^{\left(p, A_{p}\right)}\right] d x \\
=\frac{2^{\lambda-1}}{\lambda} H_{p+2, q}^{m, n+1}\left[\left.b\left(\frac{2}{-}\right)^{\sigma}\right|_{\left(b_{q}, B_{q}\right)} ^{\left(1-\frac{\lambda+v}{2}, \frac{\sigma}{2}\right),\left(p, A_{p}\right),\left(1-\frac{\lambda-v}{2}, \frac{\sigma}{2}\right)}\right] .
\end{gathered}
$$

Note the relation between Prabhakar function and the Fox- $H$ function [29]:

$$
E_{\alpha, \beta}^{\gamma}(z)=\frac{1}{\Gamma(\gamma)} H_{1,2}^{1,1}\left[-z \mid \begin{array}{l}
(1-\gamma, 1) \\
(0,1),(1-\beta, \alpha)
\end{array}\right]
$$

We define the integral operator $\mathcal{E}_{\alpha, \beta, \omega ;+}^{\gamma}$ as follows [37]:

$$
\left(\mathcal{E}_{\alpha, \beta, \omega ; a+}^{\gamma} \varphi\right)(t):=\left(t^{\beta-1} E_{\alpha, \beta}^{\gamma}\left(\omega t^{\alpha}\right)\right) * \varphi(t)=\int_{a}^{t}(t-\tau)^{\beta-1} E_{\alpha, \beta}^{\gamma}\left(\omega(t-\tau)^{\alpha}\right) \varphi(\tau) d \tau
$$

It is noted that the integral operator (11) is nowadays known in the literature as the Prabhakar fractional integral.

Lemma 1. The following Laplace transform of a Prabhakar function is given by [29,31]:

$$
L\left[t^{\beta-1} E_{\alpha, \beta}^{\gamma}\left( \pm \omega t^{\alpha}\right)\right](s)=\int_{0}^{\infty} e^{-s t} t^{\beta-1} E_{\alpha, \beta}^{\gamma}\left( \pm \omega t^{\alpha}\right) d t=\frac{s^{\alpha \gamma-\beta}}{\left(s^{\alpha} \mp \omega\right)^{\gamma}}
$$

where $\left|\omega / s^{\alpha}\right|<1$.

Lemma 2. The Laplace transform of $e^{-\kappa t} t^{\beta-1} E_{\alpha, \beta}^{\gamma}\left( \pm \omega t^{\alpha}\right)$ is given by the following formula [31]:

$$
L\left[e^{-\kappa t} t^{\beta-1} E_{\alpha \beta}^{\gamma}\left( \pm \omega t^{\alpha}\right)\right](s)=\frac{(s+\kappa)^{\alpha \gamma-\beta}}{\left((s+\kappa)^{\alpha} \mp \omega\right)^{\gamma}}
$$


where $\kappa \geq 0,\left|\omega /(s+\kappa)^{\alpha}\right|<1$.

If $\kappa=0$, then Lemma 2 coincides with Lemma 1 .

Lemma 3. For $\forall \alpha>0, \beta \in \mathbb{C}, \mu>0$ and $a \in \mathbb{R}$, the following formula is valid [39]:

$$
\int_{\mathbb{R}^{n}} e^{i \xi \cdot x} E_{\alpha, \beta}^{(m)}\left(-|\xi|^{\mu}\right) d \xi=(2 \pi)^{n / 2}|x|^{1-n / 2} \int_{0}^{\infty}|\xi|^{n / 2} E_{\alpha, \beta}^{(m)}\left(-|\xi|^{\mu}\right) J_{\frac{n}{2}-1}(|x||\xi|) d|\xi| .
$$

Here $J_{\frac{n}{2}-1}(\cdot)$ is a Bessel function and $E_{\alpha, \beta}^{(m)}(z)$ denotes $m$-th derivatives of the Mittag-Leffler function. $m$-th derivatives of the Mittag-Leffler function can be expressed in terms of the Fox H-function as

$$
E_{\alpha, \beta}^{(m)}(z)=H_{1,2}^{1,1}\left[-\left.z\right|_{(0,1),(1-(\alpha m+\beta), \alpha)} ^{(-m, 1)}\right] .
$$

For solvability of an integral equation of the Volterra type with a difference kernel the following assertion is true [40]:

Lemma 4. If $\{k(t), r(t)\} \in L_{1}[0, T]$ for a fixed $T>0$ and $k(t), r(t)$ are jointed by the integral equation

$$
r(t)=k(t)+\int_{0}^{t} k(t-\tau) r(\tau) d \tau, \quad t \in[0, T],
$$

then the solution of the integral equation

$$
\varphi(t)=\int_{0}^{t} k(t-\tau) \varphi(\tau) d \tau+f(t), \quad f(t) \in L_{1}[0, T]
$$

is given by formula

$$
\varphi(t)=\int_{0}^{t} r(t-\tau) f(\tau) d \tau+f(t)
$$

\section{Explicit Solution of the Problem (1) and (2)}

To solve problems (1) and (2), we use the Laplace and Fourier transforms of the function $u(x, t)$, respectively, $t>0, x \in \mathbb{R}^{n}$

$$
\begin{aligned}
& \hat{u}(x, s)=\int_{0}^{\infty} u(x, t) e^{-s t} d t, \quad x \in \mathbb{R}^{n}, s \in \mathbb{C}, \\
& \widetilde{u}(\xi, t):=\int_{\mathbb{R}^{n}} u(x, t) e^{-i \xi \cdot x} d x, \quad \xi \in \mathbb{R}^{n}, t>0
\end{aligned}
$$

and their inverse transformations with respect to $s \in \mathbb{C}$ and $\xi \in \mathbb{R}^{n}$

$$
\begin{gathered}
u(x, t)=L^{-1}[\hat{u}](x, s)=\frac{1}{2 \pi i} \int_{\gamma-i \infty}^{\gamma+i \infty} \hat{u}(x, s) e^{s t} d s, \quad x \in \mathbb{R}^{n}, t>0, \\
u(x, t)=F^{-1}[\widetilde{u}](\xi, t):=\frac{1}{(2 \pi)^{n}} \int_{\mathbb{R}^{n}} \widetilde{u}(\xi, t) e^{i \tau \cdot x} d \xi, \quad \xi \in \mathbb{R}^{n}, t>0 .
\end{gathered}
$$


Here, $x=\left(x_{1}, \ldots, x_{n}\right) \in \mathbb{R}^{n}, \xi=\left(\xi_{1}, \ldots, \xi_{n}\right) \in \mathbb{R}^{n}, \xi \cdot x=\sum_{j=1}^{n} \xi_{j} \cdot x_{j}, d x=d x_{1} d x_{2} \ldots d x_{n}$, $d \xi=d \xi_{1} d \xi_{2} \ldots d \xi_{n}$ and $\gamma \in \mathbb{R}$. In particular, operators (15), (17) and (16), (18) are mutually invertible on sufficiently well-behaved functions $u(x, t)$.

Let

$$
\widetilde{f}(\xi, t):=\int_{\mathbb{R}^{n}} f(x, t) e^{-i \xi \cdot x} d x, \quad \widetilde{\varphi}(\xi):=\int_{\mathbb{R}^{n}} \varphi(x) e^{-i \xi \cdot x} d x, \quad \widetilde{\psi}(\xi):=\int_{\mathbb{R}^{n}} \psi(x) e^{-i \xi \cdot x} d x .
$$

The unknown function $u(x, t)$ needs to be fairly behaved in order for it to be treated with its derivatives $u_{t}(x, t), u_{t t}(x, t), u_{x_{i} x_{i}}(x, t), i=1, \ldots, n$ by the technique of Laplace (in $t$ ) and Fourier (in $x$ ) transforms. The given functions $\varphi_{j}(x), j=0,1, \ldots, m-1$ and $f(x, t)$ are also supposed to have such properties.

Theorem 1. The analytical solution of the Equation (1) with initial and boundary conditions (2) can be expressed as the following form

$$
\begin{gathered}
u(x, t)=(2 \pi)^{-n} \int_{\mathbb{R}^{n}} \sum_{k=0}^{\infty} \frac{(-1)^{k}}{\rho^{k+1}}\left(\begin{array}{c}
\mathcal{E}^{k+1} \\
m,(m-\alpha) k+m,-\frac{|\xi|^{2}}{\rho} ; 0+
\end{array}\right)(\xi, t) e^{i \xi \cdot x} d \xi \\
+\int_{\mathbb{R}^{n}}^{m-1} \sum_{j=0}^{m-1} G_{j}(x-\xi, t) \varphi_{j}(\xi) d \xi
\end{gathered}
$$

provided that the integrals and the infinite series on the right-hand side of this equality converge, where $G_{j}(x, t), j=0, \ldots, m-1$ is the Green functions. They are given by

$$
G_{j}(x, t):=G_{1 j}(x, t)+G_{2 j}(x, t),
$$

where

$$
\begin{gathered}
G_{1 j}(x, t)=\frac{1}{2 \pi^{n / 2}|x|^{n}} \\
\times \sum_{k=0}^{\infty} \frac{(-1)^{k}}{\rho^{k} k !} t^{(m-\alpha) k+j} H_{1,2}^{2,0}\left[\frac{\sqrt{\rho}}{2} \frac{|x|}{t^{m / 2}} \mid \begin{array}{c}
(1+(m-\alpha) k+j, m / 2) \\
(n / 2,1 / 2),(1+k, 1 / 2)
\end{array}\right], \\
G_{2 j}(x, t)=\frac{1}{2 \pi^{n / 2}|x|^{n}} \\
\times \sum_{k=0}^{\infty} \frac{(-1)^{k}}{\rho^{k+1} k !} t^{(m-\alpha)(k+1)+j} H_{1,2}^{2,0}\left[\frac{\sqrt{\rho}}{2} \frac{|x|}{t^{m / 2}} \mid \begin{array}{c}
(1+(m-\alpha)(k+1)+j, m / 2) \\
(n / 2,1 / 2),(1+k, 1 / 2)
\end{array}\right] .
\end{gathered}
$$

here $x \in \mathbb{R}^{n}, \xi \in \mathbb{R}^{n}, d \xi=d \xi_{1} d \xi_{2} \ldots d \xi_{n}$.

Proof. Applyin the Laplace transforms (15) to both sides of Equation (1) with respect to variable $t$ taking into account the initial conditions from (2), the Laplace transform formula for the partial fractional derivative Caputo [23] and classical derivative

$$
L\left[{ }_{0}^{C} D_{t}^{\alpha} u\right](x, s)=s^{\alpha} \hat{u}(x, s)-\sum_{j=0}^{m-1} s^{\alpha-j-1} \frac{\partial^{j} u}{\partial t^{j}}(x, 0)
$$

and

$$
L\left[\rho \frac{\partial^{m} u}{\partial t^{m}}\right](x, s)=\rho s^{m} \hat{u}(x, s)-\sum_{j=0}^{m-1} \rho s^{m-j-1} \frac{\partial^{j} u}{\partial t^{j}}(x, 0),
$$

we obtain

$$
\rho s^{m} \hat{u}(x, s)-\sum_{j=0}^{m-1} \rho s^{m-j-1} \varphi_{j}(x)+s^{\alpha} \hat{u}(x, s)-\sum_{j=0}^{m-1} s^{\alpha-j-1} \varphi_{j}(x)-\triangle \hat{u}(x, s)=\hat{f}(x, s) .
$$


In sequence, applying to this equality the Fourier transform (16) and using well-known formula of Fourier transform of operator $\triangle$

$$
F[\triangle \hat{u}](\xi, s)=-|\xi|^{2} F[\hat{u}](\xi, s),
$$

we come to the relation

$$
\rho s^{m} \hat{\tilde{u}}(\xi, s)-\sum_{j=0}^{m-1} \rho s^{m-j-1} \tilde{\varphi}_{j}(\xi)+s^{\alpha} \hat{\tilde{u}}(\xi, s)-\sum_{j=0}^{m-1} s^{\alpha-j-1} \tilde{\varphi}_{j}(\xi)+|\xi|^{2} \hat{\tilde{u}}(\xi, s)=\hat{\tilde{f}}(\xi, s) .
$$

Equation (19) yields

$$
\hat{\tilde{u}}(\xi, s)=\frac{1}{\rho s^{m}+s^{\alpha}+|\xi|^{2}} \hat{\tilde{f}}(\xi, s)+\sum_{j=0}^{m-1} \frac{\rho s^{m-j-1}+s^{\alpha-j-1}}{\rho s^{m}+s^{\alpha}+|\xi|^{2}} \tilde{\varphi} j(\xi)=: F(\xi, s)+\sum_{j=0}^{m-1} \Phi_{j}(\xi, s) .
$$

We calculate the inverse Laplace and Fourier transforms of the function $\hat{\tilde{u}}(\xi, s)$ defined by (21). First, these operations we carry out for $F(\xi, s)$. It may be performed by using the form

$$
\frac{1}{\rho s^{m}+s^{\alpha}+|\xi|^{2}}=\frac{s^{-\alpha}}{\rho s^{m-\alpha}+1} \cdot \frac{1}{1+\frac{|\xi|^{2} s^{-\alpha}}{\rho s^{m-\alpha}+1}}
$$

and expanding the second multiplier on the right side of this term into an infinitely decreasing geometric series:

$$
\frac{1}{1+\frac{|\xi|^{2} s^{-\alpha}}{\rho s^{m-\alpha}+1}}=\sum_{n=0}^{\infty}\left(-|\xi|^{2}\right)^{n} \frac{s^{-\alpha n}}{\left(\rho s^{m-\alpha}+1\right)^{n}}
$$

for $\left|\frac{|\xi|^{2} s^{-\alpha}}{\rho s^{m-\alpha}+1}\right|<1$. On bases of (22) from the last equality we have

$$
\frac{1}{\rho s^{m}+s^{\alpha}+|\xi|^{2}}=\frac{1}{\rho} \sum_{n=0}^{\infty}\left(-\frac{1}{\rho}|\xi|^{2}\right)^{n} \frac{s^{-\alpha(n+1)}}{\left(s^{m-\alpha}+\frac{1}{\rho}\right)^{n+1}} .
$$

Then, according to Lemma 1, we note

$$
\frac{s^{-\alpha(n+1)}}{\left(s^{m-\alpha}+\frac{1}{\rho}\right)^{(n+1)}}=L\left[t^{m(n+1)-1} E_{m-\alpha, m(n+1)}^{n+1}\left(-\frac{t^{m-\alpha}}{\rho}\right)\right]
$$

and

$$
\frac{1}{\rho s^{m}+s^{\alpha}+|\xi|^{2}}=L\left[\frac{1}{\rho} \sum_{n=0}^{\infty}\left(-\frac{1}{\rho}|\xi|^{2}\right)^{n} t^{m(n+1)-1} E_{m-\alpha, m(n+1)}^{n+1}\left(-\frac{t^{m-\alpha}}{\rho}\right)\right] .
$$

Taking these formulae into account, eventually $F(\xi, s)$ can be expressed as

$$
F(\xi, s)=L\left[\frac{1}{\rho} \sum_{n=0}^{\infty}\left(-\frac{1}{\rho}|\xi|^{2}\right)^{n} t^{m(n+1)-1} E_{m-\alpha, m(n+1)}^{n+1}\left(-\frac{t^{m-\alpha}}{\rho}\right)\right] L[\tilde{f}(\xi, t)] .
$$

Now, we apply the inverse transform the functions $\Phi_{j}(\xi, s), j=0,1, \ldots, m-1$. For this, we note that the fractions at the functions $\varphi_{j}(\xi), j=0,1, \ldots, m-1$ in expressions for $\Phi_{j}(\xi, s)$ as seen from (21) differ from (22) only by numerators. Because of this for fixed $j$ we have

$$
\frac{\rho s^{m-j-1}+s^{\alpha-j-1}}{\rho s^{m}+s^{\alpha}+|\xi|^{2}}=\sum_{n=0}^{\infty}\left(-\frac{1}{\rho}|\xi|^{2}\right)^{n}\left[\frac{s^{m-\alpha(n+1)-j-1}}{\left(s^{m-\alpha}+\frac{1}{\rho}\right)^{n+1}}+\frac{1}{\rho} \cdot \frac{s^{-\alpha n-j-1}}{\left(s^{m-\alpha}+\frac{1}{\rho}\right)^{n+1}}\right],
$$


In view of the last relations, applying Lemma 1 to the frictions in the definition of $\Phi_{j}(\xi, s)$, we obtain

$$
\begin{array}{r}
\Phi_{j}(\xi, s)=L\left[\sum_{n=0}^{\infty}\left(-\frac{1}{\rho}|\xi|^{2}\right)^{n} t^{m n+j} E_{m-\alpha, m n+j+1}^{n+1}\left(-\frac{t^{m-\alpha}}{\rho}\right)\right](s) \tilde{\varphi}_{j}(\xi) \\
+L\left[\frac{1}{\rho} \sum_{n=0}^{\infty}\left(-\frac{1}{\rho}|\xi|^{2}\right)^{n} t^{m(n+1)-\alpha+j} E_{m-\alpha, m(n+1)-\alpha+j+1}^{n+1}\left(-\frac{t^{m-\alpha}}{\rho}\right)\right](s) \tilde{\varphi}_{j}(\xi),
\end{array}
$$

$j=0, \ldots, m-1$.

Further, in accordance with the Prabhakar function definition (3), from Equation (23) we obtain

$$
\begin{gathered}
\frac{1}{\rho} \sum_{n=0}^{\infty}\left(-\frac{1}{\rho}|\xi|^{2}\right)^{n} t^{m(n+1)-1} E_{m-\alpha, m(n+1)}^{n+1}\left(-\frac{t^{m-\alpha}}{\rho}\right) \\
=\frac{1}{\rho} \sum_{n=0}^{\infty}\left(-\frac{1}{\rho}|\xi|^{2}\right)^{n} t^{m(n+1)-1} \sum_{k=0}^{\infty} \frac{(n+1)_{k}}{\Gamma((m-\alpha) k+m(n+1))} \frac{\left(-\frac{t^{m-\alpha}}{\rho}\right)^{k}}{k !} \\
=\sum_{n=0}^{\infty} \sum_{k=0}^{\infty} \frac{(-1)^{k}}{\rho^{k+1}} t^{(m-\alpha) k+m-1} \frac{(k+1)_{n}}{\Gamma((m-\alpha) k+m(n+1))} \frac{\left(-\frac{t^{m}}{\rho}|\xi|^{2}\right)^{n}}{n !} \\
=\sum_{k=0}^{\infty} \frac{(-1)^{k}}{\rho^{k+1}} t^{(m-\alpha) k+m-1} E_{m,(m-\alpha) k+m}^{k+1}\left(-\frac{t^{m}}{\rho}|\xi|^{2}\right) .
\end{gathered}
$$

By virtue of this fact we continue converting the function $F(\xi, s)$ into

$$
\begin{gathered}
F(\xi, s)=L\left[\sum_{k=0}^{\infty} \frac{(-1)^{k}}{\rho^{k+1}} t^{(m-\alpha) k+m-1} E_{m,(m-\alpha) k+m}^{k+1}\left(-\frac{t^{m}}{\rho}|\xi|^{2}\right)\right] \\
\times L[\tilde{f}(\xi, t)]=L\left[\left(\sum_{k=0}^{\infty} \frac{(-1)^{k}}{\rho^{k+1}} t^{(m-\alpha) k+m-1} E_{m,(m-\alpha) k+m}^{k+1}\left(-\frac{t^{m}}{\rho}|\xi|^{2}\right)\right) * \tilde{f}(\xi, t)\right] .
\end{gathered}
$$

According to the convolution property of the Laplace transform and the definition of integral operator $\mathcal{E}_{\alpha, \beta, \omega ;+}^{\gamma} \varphi$ by (11), the inverse Laplace transform of the function $F(\xi, s)$ from last relations can be obtained as follows:

$$
L^{-1}[F(\xi, s)](t)=\sum_{k=0}^{\infty} \frac{(-1)^{k}}{\rho^{k+1}}\left(\begin{array}{l}
\mathcal{E}^{k+1} \\
m,(m-\alpha) k+m,-\frac{|\xi|^{2}}{\rho} ; 0+
\end{array} \tilde{f}\right)(\xi, t) .
$$

Analogically, the inverse Laplace transform of functions $\Phi_{j}(\xi, s)$ can be expressed as

$$
\begin{gathered}
L^{-1}\left[\Phi_{j}(\xi, s)\right](t)=\sum_{k=0}^{\infty} \frac{(-1)^{k}}{\rho^{k}} t^{(m-\alpha) k+j} E_{m,(m-\alpha) k+j+1}^{k+1}\left(-\frac{t^{m}}{\rho}|\xi|^{2}\right) \tilde{\varphi}_{j}(\xi) \\
+\sum_{k=0}^{\infty} \frac{(-1)^{k}}{\rho^{k+1}} t^{(m-\alpha)(k+1)+j} E_{m,(m-\alpha)(k+1)+j+1}^{k+1}\left(-\frac{t^{m}}{\rho}|\xi|^{2}\right) \tilde{\varphi}_{j}(\xi), j=0,1, \ldots, m-1 .
\end{gathered}
$$

Considering the relationship between the generalized Mittag-Leffler function and the Fox $H$ function (10), the last equalities can be rewritten in the form [29]

$$
\begin{gathered}
L^{-1}\left[\Phi_{j}(\xi, s)\right](t)=\sum_{k=0}^{\infty} \frac{(-1)^{k}}{\rho^{k}} t^{(m-\alpha) k+j} \frac{1}{\Gamma(k+1)} H_{1,2}^{1,1}\left[\frac{t^{m}}{\rho}|\xi|^{2} \mid \begin{array}{l}
(-k, 1) \\
(0,1),(-(m-\alpha) k-j, m)
\end{array}\right] \tilde{\varphi}_{j}(\xi) \\
+\sum_{k=0}^{\infty} \frac{(-1)^{k}}{\rho^{k+1}} t^{(m-\alpha)(k+1)+j} \frac{1}{\Gamma(k+1)} H_{1,2}^{1,1}\left[\frac{t^{m}}{\rho}|\xi|^{2} \mid \begin{array}{l}
(-k, 1) \\
(0,1),(-(m-\alpha)(k+1)-j, m)
\end{array}\right] \tilde{\varphi}_{j}(\xi)
\end{gathered}
$$




$$
\begin{gathered}
=\sum_{k=0}^{\infty} \frac{(-1)^{k}}{\rho^{k} k !}\left(t^{(m-\alpha) k+j} H_{1,2}^{1,1}\left[\left.\frac{t^{m}}{\rho}|\xi|^{2}\right|_{(0,1),(-(m-\alpha) k-j, m)} ^{(-k, 1)}\right]\right. \\
\left.+\frac{t^{(m-\alpha)(k+1)+j}}{\rho} H_{1,2}^{1,1}\left[\left.\frac{t^{m}}{\rho}|\xi|^{2}\right|_{(0,1),(-(m-\alpha)(k+1)-j, m)} ^{(-k, 1)}\right]\right) \tilde{\varphi}(\xi) \\
=: \sum_{k=0}^{\infty} \frac{(-1)^{k}}{\rho^{k} k !}\left(t^{(m-\alpha) k+j} \tilde{\Phi}_{j k}^{(1)}(\xi, t)+\frac{t^{(m-\alpha)(k+1)+j}}{\rho} \tilde{\Phi}_{j k}^{(2)}(\xi, t)\right) \tilde{\varphi}(\xi), j=0,1, \ldots, m-1 .
\end{gathered}
$$

In (27) we introduced the following notations:

$$
\begin{gathered}
\tilde{\Phi}_{j k}^{(1)}(\xi, t)=H_{1,2}^{1,1}\left[\left.\frac{t^{m}}{\rho}|\xi|^{2}\right|_{(0,1),(-(m-\alpha) k-j, m)} ^{(-k, 1)}\right], \\
\tilde{\Phi}_{j k}^{(2)}(\xi, t)=H_{1,2}^{1,1}\left[\left.\frac{t^{m}}{\rho}|\xi|^{2}\right|_{(0,1),(-(m-\alpha)(k+1)-j, m)} ^{(-k, 1)}\right], j=0,1, \ldots, m-1, k=0,1,2, \ldots
\end{gathered}
$$

Now we compute the inverse Fourier transform of relations (27). Equation (28) can be further manipulated by employing an inverse Fourier transform; we have

$$
\begin{gathered}
\Phi_{j k}^{(1)}(x, t)=\frac{1}{(2 \pi)^{n}} \int_{\mathbb{R}^{n}} H_{1,2}^{1,1}\left[\left.\frac{t^{m}}{\rho}|\xi|^{2}\right|_{(0,1),(-(m-\alpha) k-j, m)} ^{(-k, 1)}\right] e^{i \xi \cdot x} d \xi, \\
\Phi_{j k}^{(2)}(x, t)=\frac{1}{(2 \pi)^{n}} \int_{\mathbb{R}^{n}} H_{1,2}^{1,}\left[\left.\frac{t^{m}}{\rho}|\xi|^{2}\right|_{(0,1),(-(m-\alpha)(k+1)-j, m)} ^{(-k, 1)}\right] e^{i \xi \cdot x} d \xi,
\end{gathered}
$$

here $j=0,1, \ldots, m-1, k=0,1,2, \ldots$. Using Lemma 3, we obtain the following results from formulae (29) and (30)

$$
\begin{gathered}
\Phi_{j k}^{(1)}(x, t)=\frac{1}{(2 \pi)^{n / 2}}|x|^{1-\frac{n}{2}} \int_{0}^{\infty} y^{n / 2} H_{1,2}^{1,1}\left[\left.\frac{t^{m}}{\rho} y^{2}\right|_{(0,1),(-(m-\alpha) k-j, m)} ^{(-k, 1)}\right] \mathfrak{J}_{\frac{n}{2}-1}(|x| y) d y, \\
\Phi_{j k}^{(2)}(x, t)=\frac{1}{(2 \pi)^{n / 2}}|x|^{1-\frac{n}{2}} \int_{0}^{\infty} y^{n / 2} H_{1,2}^{1,1}\left[\left.\frac{t^{m}}{\rho} y^{2}\right|_{(0,1),(-(m-\alpha)(k+1)-j, m)} ^{(-k, 1)}\right] \mathfrak{J}_{\frac{n}{2}-1}(|x| y) d y, \\
j=0,1, \ldots, m-1, k=0,1,2, \ldots
\end{gathered}
$$

Taking into account (9) and the properties Fox's $H$-function (6)-(8), the first function of the last ones can be written as

$$
\begin{aligned}
\Phi_{1 j}(x, t)=\frac{1}{\pi^{n / 2}|x|^{n}} H_{3,2}^{1,2}\left[\frac{4 t^{m}}{\rho|x|^{2}} \mid \begin{array}{c}
\left(1-\frac{n}{2}, 1\right),(-k, 1),(0,1) \\
(0,1),(-(m-\alpha) k-j, m)
\end{array}\right] \\
=\frac{1}{\pi^{n / 2}|x|^{n}} H_{2,1}^{0,2}\left[\frac{4 t^{m}}{\rho|x|^{2}} \mid \begin{array}{c}
\left(1-\frac{n}{2}, 1\right),(-k, 1) \\
(-(m-\alpha) k-j, m)
\end{array}\right] \\
=\frac{1}{\pi^{n / 2}|x|^{n}} H_{1,2}^{2,0}\left[\frac{\rho|x|^{2}}{4 t^{m}} \mid \begin{array}{c}
(1+(m-\alpha) k+j, m) \\
(n / 2,1),(1+k, 1)
\end{array}\right] \\
=\frac{1}{2 \pi^{n / 2}|x|^{n}} H_{1,2}^{2,0}\left[\frac{\sqrt{\rho}}{2} \frac{|x|}{t^{m / 2}} \mid \begin{array}{c}
(1+(m-\alpha) k+j, m / 2) \\
(n / 2,1 / 2),(1+k, 1 / 2)
\end{array}\right] .
\end{aligned}
$$

By same argument for $\Phi_{2 j}$ we have

$$
\Phi_{2 j}(x, t)=\frac{1}{2 \pi^{n / 2}|x|^{n}} H_{1,2}^{2,0}\left[\frac{\sqrt{\rho}}{2} \frac{|x|}{t^{m / 2}} \mid \begin{array}{c}
(1+(m-\alpha)(k+1)+j, m / 2) \\
(n / 2,1 / 2),(1+k, 1 / 2)
\end{array}\right] .
$$


Now, applying the inverse Fourier transform to both sides of (26) and (27), and substituting into the resulting equalities, formulae (31) and (32), we obtain

$$
G_{j}(x, t):=G_{1 j}(x, t)+G_{2 j}(x, t),
$$

where

$$
\begin{gathered}
G_{1 j}(x, t)=\frac{1}{2 \pi^{n / 2}|x|^{n}} \times \\
\times \sum_{k=0}^{\infty} \frac{(-1)^{k}}{\rho^{k} k !} t^{(m-\alpha) k+j} H_{1,2}^{2,0}\left[\frac{\sqrt{\rho}}{2} \frac{|x|}{t^{m / 2}} \mid \begin{array}{c}
(1+(m-\alpha) k+j, m / 2) \\
(n / 2,1 / 2),(1+k, 1 / 2)
\end{array}\right], \\
G_{2 j}(x, t)=\frac{1}{2 \pi^{n / 2}|x|^{n}} \\
\times \sum_{k=0}^{\infty} \frac{(-1)^{k}}{\rho^{k+1} k !} t^{(m-\alpha)(k+1)+j} H_{1,2}^{2,0}\left[\frac{\sqrt{\rho}}{2} \frac{|x|}{t^{m / 2}} \mid \begin{array}{c}
(1+(m-\alpha)(k+1)+j, m / 2) \\
(n / 2,1 / 2),(1+k, 1 / 2)
\end{array}\right] .
\end{gathered}
$$

Continuing to convert the equalities (26) and (27) we can write formally

$$
L^{-1}\left(\Phi_{j}(\xi, s)\right)=L^{-1}\left(F\left[G_{j}(x, s)\right](\xi) \tilde{\varphi}_{j}(\xi)\right) .
$$

In view of (27) and (34), employing an inverse Laplace transform to Equation (21) we finally obtain

$$
\tilde{u}(\xi, t)=\sum_{k=0}^{\infty} \frac{(-1)^{k}}{\rho^{k+1}}\left(\begin{array}{l}
\mathcal{E}_{m,(m-\alpha) k+m,-\frac{|\xi|^{2}}{\rho} ; 0+}^{k+1} \\
\tilde{f}
\end{array}\right)(\xi, t)+\sum_{j=0}^{m-1} F\left[G_{j}(x, t)\right](\xi) \varphi_{j}(\xi) .
$$

To Equation (35) can be further applied inverse the Fourier transform and Fourier convolution property one by one. Therefore, we complete the proof of Theorem 1 .

\section{The Integro-Differential Diffusion Equation with the Mittag-Leffler Type Kernel}

In this section we demonstrate the equivalence of one integro-differential wave equation with the Mittag-Leffler type kernel to the fractional wave equation.

Theorem 2. The integro-differential wave equation

$$
\rho \frac{\partial^{m} u}{\partial t^{m}}-\triangle u+\int_{0}^{t} k(t-\tau) \triangle u(x, \tau) d \tau=0, x \in \mathbb{R}^{n}, t>0
$$

with memory $k(t)=t^{m-1-\alpha} E_{m-\alpha, m-\alpha}\left(-t^{m-\alpha}\right), m-1<\alpha \leq m$, is equivalent to the timefractional wave equation

$$
\frac{\partial^{m} u}{\partial t^{m}}+{ }_{0}^{C} D_{t}^{\alpha} u-\frac{1}{\rho} \triangle u(x, t)=0 .
$$

Proof. In general the Equation (36) is the Volterra integral equation of the second kind with respect to $\Delta u$ for fixed $x$ and employing Lemma 4 , we obtain

$$
\frac{1}{\rho} \triangle u=\frac{\partial^{m} u}{\partial t^{m}}+\int_{0}^{t} r(t-\tau) \frac{\partial^{m} u}{\partial \tau^{m}}(x, \tau) d \tau,
$$

where $r(t)$ is resolvent of $k(t)$ and it satisfies the integral Equation (5).

We use to both sides of (12) the Laplace and denoting by $K(s)$ and $R(s)$ the imagines of origins $k(t)$ and $r(t)$, respectively, we have

$$
R(s)=K(s)+K(s) R(s) .
$$


From this relation we obtain

$$
K(s)=\frac{R(s)}{1+R(s)}=\frac{1}{s^{m-\alpha}+1}, \mathfrak{R}(s)>1 .
$$

Applying the inverse Laplace transformation to last equality (see [33])

$$
k(t)=L^{-1}[K(s)]=L^{-1}\left[\frac{1}{s^{m-\alpha}+1}\right]=t^{m-1-\alpha} E_{m-\alpha, m-\alpha}\left(-t^{m-\alpha}\right) .
$$

Hence, if we suppose that the function $k(t)$ is given by formula (39) then, (38) hlyields (37).

Remark 1. The Equation (36) with memory kernel $k(t)=t^{m-1-\alpha} E_{m-\alpha, m-\alpha}\left(-t^{m-\alpha}\right)$ describes the time-fractional wave equation.

From this comment it follows that the solution of Equation (36) with initial-boundary condition can be given by formula (16) for $f(x, t)=0$.

\section{Conclusions}

Equation (36) (with different types of memory kernels) can be used to investigate a wide class of non-classical mathematical models in which the memory effect is present. In this paper, we show that the time-fractional multidimensional differential Equation (1) can be obtained from Equation (36) with the Mittag-Leffler kernel $t^{m-1-\alpha} E_{m-\alpha, m-\alpha}\left(-t^{m-\alpha}\right)$. Applying the method of the Laplace transform in the time variable and the Fourier transform in the spatial variable, an explicit solution of problem (1) and (2) is obtained, which includes the fractional Prabhakar integral and the Fox $\mathrm{H}$ - functions. In many cases, due to the nonlocality of the integral term, it is not possible to obtain an exact solution to Equation (1). Therefore, in many cases, a more reasonable option is to find its numerical solution. In the future, we plan to numerically solve similar problems in one-dimensional and two-dimensional cases.

Author Contributions: Conceptualization, M.A.S. and D.K.D.; methodology, M.A.S. and D.K.D.; validation, D.K.D. and A.A.R.; formal analysis, D.K.D. and A.A.R.; investigation, M.A.S., D.K.D. and A.A.R.; resources, D.K.D. and A.A.R.; writing—original draft preparation, M.A.S. and D.K.D.; writing-review and editing, D.K.D. and A.A.R.; supervision, D.K.D.; project administration, A.A.R.; funding acquisition, M.A.S. All authors have read and agreed to the published version of the manuscript.

Funding: The first author (M.A.S.) was financially supported by the Ministry of Education and Science of the Republic of Kazakhstan (project AP09258836).

Conflicts of Interest: The authors declare no conflict of interest.

\section{References}

1. Boltzmann, L. Zur theorie der elastischen Nachwirkung. Ann. Der Phys. 1878, 241, 430-432. [CrossRef]

2. Vronsky, A.P. The phenomenon of aftereffect in à solid. Acad. Sci. Ussr. Appl. Math. Mech. 1941, 5, 31-56. (In Russian)

3. Gerasimov, A.N. Generalization of linear deformation laws and their application to problems of internal friction. Acad. Sci. Ussr. Appl. Math. Mech. 1948, 12, 529-539. (In Russian)

4. Tarasova, V.V.; Tarasov V.E. Economic Interpretation of Fractional Derivatives. Progr. Fract. Differ. Appl. 2017, 3, 1-6. [CrossRef]

5. Uchaikin, V.V. Background and Theory. In Fractional Derivatives for Physicists and Engineers; Springer: Berlin/Heidelberg, Germany, 2013; p. 373.

6. Volterra, V. Sur les equations integro-differentielles et leurs applications. Acta Math. 1912, 35, 295-356. [CrossRef]

7. Colombo, F.; Guidetti, D. Some results on the identification of memory kernels. In Modern Aspects of the Theory of Partial Differential Equations; Ruzhansky, M., Wirth, J., Eds.; Springer: Basel, Switzerland, 2011; pp. 121-138.

8. Durdiev, D.K. The inverse problem of determining two coefficients in an integrodifferential wave equation. Sib. Zh. Ind. Mat. 2009, 12, 28-40. (In Russian)

9. Durdiev, D.K. An identification problem of memory function of à medium and the form of an impulse source. J. Sib. Fed. Univ. Math. Phys. 2009, 2, 127-136. 
10. Janno, J.; Von Welfersdorf, L. Inverse problems for identification of memory kernels in viscoelasticity. Math. Methods Appl. Sci. 1997, 20, 291-314. [CrossRef]

11. Durdiev, D.Q.; Totieva, Z.D. The problem of determining the multidimensional kernel of viscoelasticity equation. Vladikavkaz. Mat. Zh. 2015, 17, 18-43. (In Russian)

12. Durdiev D.K.; Safarov, Z.S. Inverse problem of determining the one-dimensional kernel of the viscoelasticity equation in à bounded domain. Math. Notes 2015, 97, 867-877. [CrossRef]

13. Durdiev, D.K.; Totieva, Z.D. The problem of determining the one-dimensional kernel of the viscoelasticity equation. Sib. Zh. Ind. Mat. 2013 16, 72-82. (In Russian)

14. Durdiev, D.K. Inverse problem for the identification of à memory kernel from Maxwell's system integrodifferential equations for à homogeneous anisotropic media. Nanosyst. Phys. Chem. Math. 2015, 6, 268-273. [CrossRef]

15. Durdiev, D.K.; Rahmonov, A.A. A 2D kernel determination problem in à visco-elastic porous medium with à weakly horizontally inhomogeneity. Math. Methods Appl. Sci. 2020, 43, 8776-8796. [CrossRef]

16. Durdiev, D.K.; Rakhmonov, A.A. Inverse problem for à system of integro-differential equations for $\mathrm{SH}$ waves in à visco-elastic porous medium: Global solvability. Theor. Math. Phys. 2018, 195, 923-937. [CrossRef]

17. Durdiev, D.K.; Rahmonov, A.A. The problem of determining the $2 \mathrm{D}-$ kernel in à system of integrodifferential equations of à viscoelastic porous medium. Jur. Appl. Industr. Math. 2020, 14, 281-295. [CrossRef]

18. Durdiev, U.D. A problem of identification of à special 2D memory kernel in an integro-differential hyperbolic equation. Eurasian J. Math. Comput. Appl. 2019, 7, 4-19.

19. Durdiev, U.; Totieva, Z. A problem of determining à special spatial part of 3D memory kernel in an integrodifferential hyperbolic equation. Math. Methods Appl. Sci. 2019, 42, 7440-7451. [CrossRef]

20. Rahmonov, A.A.; Durdiev, U.D.; Bozorov, Z.R. Problem of determining the speed of sound and the memory of an anisotropic medium. Theor. Math. Phys. 2021, 207, 494-513. [CrossRef]

21. Durdiev D.K.; Zhumaev Z.Z. Problem of determining à multidimensional thermal memory in à heat conductivity equation. Methods Funct. Anal. Topol. 2019, 25, 219-226.

22. Durdiev, D.K.; Zhumaev, Z.Z. Problem of determining the thermal memory of à conducting medium. Differ. Equ. 2020, 56, 785-796. [CrossRef]

23. Kilbas, A.A.; Srivastava, H.M. and Trujillo, J.J. Theory and Applications of Fractional Differential Equations; Elsevier: Amsterdam, The Netherlands, 2006; p. 523.

24. Meilanov, R.P.; Yanpolov, M.S. Features of the Phase Trajectory of à Fractal Oscillator. Tech. Phys. Lett. 2002, 28, 30-32. [CrossRef]

25. Mainardi, F.; Luchko, Y.; Pagnini, G. The fundamental solution of the space-time fractional diffusion equation. Fract. Calc. Appl. Anal. 2001, 4, 153-192.

26. Aghajani, A.; Jalilian, Y.; Trujillo, J.J. On the existence of solutions of fractional integro-differential equations. Fract. Calc. Appl. Anal. 2012, 15, 44-69. [CrossRef]

27. Schumer, R.; Benson, D.A.; Meerschaert, M.M.; Baeumer, B. Fractal mobile/immobile solute transport. Water Resour. Res. 2003, 39, 1-12. [CrossRef]

28. Giusti, A.; Colombaro, I.; Garra, R.; Garrappa, R.; Polito, F.; Popolizio, M.; Mainardi, F. A practical guide to Prabhakar fractional calculus. Fract. Calc. Appl. Anal. 2020, 23, 9-54. [CrossRef]

29. Mathai, A.M.; Saxena, R.K.; Haubold, H.J. The H-Function: Theory and Application; Springer: Berlin/Heidelberg, Germany, $2010 ; 268 p$.

30. Tomovski, Z̆.; Sandev, T. Effects of à fractional friction with power-law memory kernel on string fibrations. Comput. Math. Appl. 2011, 62, 1554-1561. [CrossRef]

31. Tomovski, Z̆.; Hilfer, R.; Srivastava, H.M. Fractional and operational calculus with generalized fractional derivatives operators and Mittag-Leffler type functions. Integral Transform. Spec. Funct. 2010, 21, 797-814. [CrossRef]

32. Li, C.; Deng, W.H.; Shen, X.Q. Exact solutions and the asymptotic behaviors for the averaged generalized fractional elastic models. Commun. Theor. Phys., 2014, 62, 443-450. [CrossRef]

33. Zao, Y.; Zao, F. The analytical solution of parabolic Volterra integro-differential equations in the infinite domain. Entropy 2016, 18, 14.

34. Hille, E.; Tamarkin, J.D. On the theory of linear integral equations. Ann. Math. 1930, 31, 479-528. [CrossRef]

35. Luchko, Y. Initial-boundary-value problems for the one-dimensional time-fractional diffusion equation. Fract. Calc. Appl. Anal. 2012, 15, 141-160. [CrossRef]

36. Gorenflo, R.; Mainardi, F. Fractional calculus: Integral and differential equations of fractional order. In Fractals and Fractional Calculus in Continuum Mechanics; Springer: Wien, Austria; New York, NY, USA, 1997; pp. 223-276.

37. Haubold, H.J.; Mathai, A.M.; Saxena, R.K. Mittag-Leffl functions and their applications. Jur. App. Math. $2011,2011,51$.

38. Fox, C. The $G$ and $H$ functions and as symmetrical Fourier kernels. Trans Amer Math Soc. 1961, 98, 395-429.

39. Zhang, Y.; Benson, D.A.; Reeves, D.M. Time and space nonlocalities underlying fractional-derivative models: Distinction and literature review of field applications. Adv. Water Resour. 2009, 32, 561-581. [CrossRef]

40. Durdiev, D.K.; Shishkina, E.L.; Sitnik S.M. The explicit formula for solution of anomalous diffusion equation in the multidimensional space. Lobacherskii J. Mat. 2021, 42, 1264-1273. [CrossRef] 